\title{
Scelte di Portafoglio: Approccio Media-Varianza Stati di Natura Continui (Mean-Variance Portfolio Choice)
}

\author{
Maria Augusta Miceli \\ Department of Economics and Law \\ University of Rome "Sapienza" \\ IFIR
}

April 7, 2019

\begin{abstract}
L'obiettivo di queste note è evidenziare il metodo di ottimizzazione del portafoglio unico per cui l'investitore massimizza la sua funzione di utilità in media e varianza del portafoglio rispetto al vincolo della frontiera efficiente dei possibili pesi in cui le attività finanziarie disponibili sono detenute nel portafoglio. Tale frontiera sarà una retta, la "capital market line", se esiste un'attività risk-free o una curva se vi sono solo attività rischiose. Il CAPM non è altro che il primo caso, ma dove la CML è tangente alla frontiera efficiente delle attività rischiose. Essendo tale punto il portafoglio dominante dovrà essere l'indice di mercato. Tale tangenza definisce i "beta" come i coefficienti di regressione del rendimento di un'attività finanziaria qualunque verso l'eccesso di rischio presento sul mercato. Di conseguenza tale regressione calcola il rendimento medio atteso per un'attività finanziaria che abbia correlazione "beta" verso il rischio di mercato.
\end{abstract}

\begin{abstract}
The aim of these notes is to highlight the method of portfolio optimization, when the investor maximizes his mean-variance utility function subject to the constraint represented by the efficient frontier of the possible weights in which the available financial assets available are held. This frontier will be a straight line, the "capital market line", when there is a risk-free asset or a curve when there are only risky assets. The CAPM is nothing else than the first case, but where the CML is tangent to the efficient frontier of risky activities. Since the tangency condition defines the dominant portfolio, this must be the market index. This tangency defines also the "betas" as the regression coefficients of the return of any financial asset towards the excess risk present on the market. Consequently, this regression calculates the expected average return for a financial asset that has a "beta" correlation with market risk.
\end{abstract}

KEYWORDS: portafoglio, portfolio, asset pricing, mean-variance, media-varianza, CAPM, efficient frontier,

JEL: A22, A23, D53, G11, G12. 


\section{Introduzione}

L'Approccio media-varianza è utile perché permette di utilizzare

Ipotesi:

1. Stati di natura infiniti.

2. Distribuzione di probabilità definita dai soli primi due momenti: media $\mu$ e varianza $\sigma^{2}$.

L'obiettivo è ricondurre la scelta di portafoglio di un investitore al modello di scelta vincolata standard.

In questo contesto:

1. la funzione obiettivo è una funzione di utilità rispetto al rendimento del portafoglio;

2. gli oggetti di scelta sono i "pesi" che le diverse attività finanziarie disponibili, di cui sono note le distribuzioni dei rendimenti, vengono tenuti in portafoglio, in modo da massimizzare il rendimento del portafoglio, dato un rischio desiderato espresso dalla variazione standard, oppure in modo da minimizzare il rischio, per un rendimento desiderato, dato.

Allo scopo di rendere il problema matematicamente "trattabile" si considerano variabili tali per cui i primi due momenti della distribuzione (media e varianza) siano condizioni sufficienti per la descrizione delle distribuzioni delle variabili aleatorie.

I dati del problema sono:

- una funzione di utilità derivabile espressa nel rendimento del portafoglio, sintetizzata nei primi due momenti, media e varianza del portafoglio,

- la distribuzione dei rendimenti delle attività finanziarie

$$
\widetilde{\mathbf{r}} \sim N(\boldsymbol{\mu}, \boldsymbol{\Sigma})
$$

esplicitando la forma matriciale per $K=2$, attività finanziarie

$$
\left(\begin{array}{c}
\widetilde{r}_{1} \\
\widetilde{r}_{2}
\end{array}\right) \sim N\left[\left(\begin{array}{l}
\mu_{1} \\
\mu_{2}
\end{array}\right),\left(\begin{array}{ll}
\sigma_{1}^{2} & \sigma_{12} \\
\sigma_{21} & \sigma_{2}^{2}
\end{array}\right)\right]
$$

La matrice delle varianze e covarianze, $\boldsymbol{\Sigma}$, può anche essere espressa mediante il coefficiente di correlazione

$$
\sigma_{i j}=\rho_{i j} \sigma_{i} \sigma_{j}
$$

ovvero

$$
\boldsymbol{\Sigma}=\left(\begin{array}{ll}
\sigma_{1}^{2} & \rho_{12} \sigma_{1} \sigma_{2} \\
\rho_{21} \sigma_{1} \sigma_{2} & \sigma_{2}^{2}
\end{array}\right)
$$

dove i due termini diagonali sono identici.

Proposizione 1 Conoscere $i$ soli primi due momenti della distribuzione è sufficiente se vale una delle seguenti condizioni

(i) la distribuzione dei rendimenti è arbitraria, ma la f. utilità è quadratica, ovvero la funzione di utilità consideri soltanto $i$ primi due momenti (media e varianza),

(ii) la funzione di utilità sia arbitraria, ma i rendimenti siano distribuiti normalmente e pertanto i primi due momenti ne descrivano completamente la distribuzione.

Nell'approccio semplificato che seguiamo, entrambe le condizioni (i) e (ii) saranno utilizzate entrambe. 


\section{Metodo di scelta}

Proprietà di diverse forme di funzioni di utilità:

1. f. utilità quadratica.

Pro: media e varianza sufficienti.

Contro: $\exists$ sazietà; coefficiente di avversione al rischio assoluto (ARA) crescente. Entrambi portano a risultati controntuitivi.

2. f. utilità normale.

Pro: media e varianza sufficienti.

Contro: il dominio va fino a $-\infty$ e più portare a consumo negativo.

3. f. lognormale.

Contro: non è preservata con l'additività.

Nel seguito utilizzaremo la funzione di utilità quadratica nei confronti di una ricchezza data dal portafoglio $\widetilde{r_{P}}$, per sua natura aleatoria perché dipende dall'andamento di rendimenti aleatori. essendo una combinazione lineare, con pesi $w_{k}, k=1, \ldots, K$ dei rendimenti aleatori delle attività finanziarie.

$$
\widetilde{r}_{P}=\sum_{k=1}^{K} w_{k} \widetilde{r}_{k}
$$

La funzione di utilità espressa nella ricchezza aleatoria è una espansione in serie di Taylor fino al secondo ordine intorno al punto definito dal valore atteso della ricchezza $E\left(\widetilde{r}_{P}\right)$.

$$
\begin{gathered}
u\left(\widetilde{r}_{P}\right)=a E\left(\widetilde{r}_{P}\right)+b\left[\widetilde{r}_{P}-E\left(\widetilde{r}_{P}\right)\right]^{2} \\
u\left(\widetilde{r}_{P}\right)=a \mu_{p}+b \sigma_{p}^{2}
\end{gathered}
$$

Nel seguito diamo qualche dettaglio in più sugli ingredienti accennati. In questa versione linearizzata e semplificata, il segno davanti al coefficiente $b$ definisce l'attitudine verso il rischio dell'investitore.

\section{Agente avverso al rischio (Risk averse)}

Il rendimento medio è un "bene" mentre la volatilità, espressa dalla varianza, è un "male" ed ha segno negativo.

$$
u\left(\widetilde{r}_{P}\right)=a \mu_{P}-b \sigma_{P}^{2}
$$

Calcoliamo il differenziale totale, per poter calcolare il SMS

$$
d U=a d \mu_{p}-\left(2 b \sigma_{p}\right) d \sigma_{p}=0
$$

da cui

$$
S M S \equiv \frac{d \mu_{p}}{d \sigma_{p}}=\frac{2 b \sigma_{p}}{a}>0
$$

Disegnamo la curva d'indifferenza $a \mu_{p}-b \sigma_{p}^{2}=\bar{u}$, fissando i parametri $a=5, b=5, \bar{u}=4$, 8 , e ricavando $\mu_{p}$ in funzione di $\sigma_{p}^{2}$.

$$
\begin{aligned}
a \mu_{p}-b \sigma_{p}^{2} & =\bar{u} \Longrightarrow \\
\mu_{p} & =4+\frac{5 x^{2}}{5} \text { e } \mu_{p}=8+\frac{5 x^{2}}{5}
\end{aligned}
$$




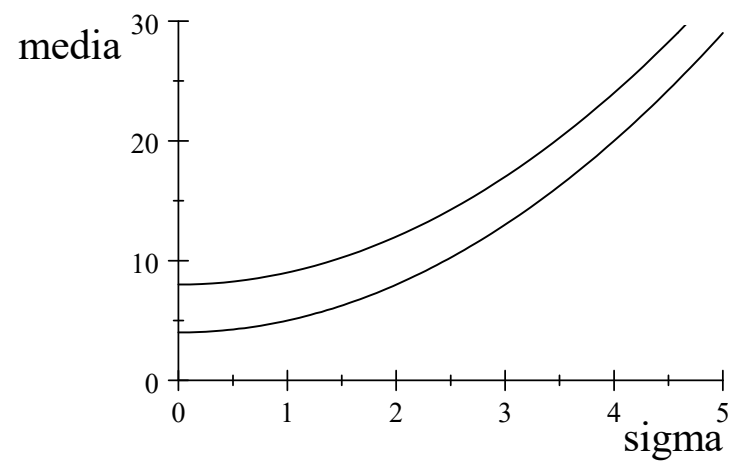

l'utilità cresce nella direzione verso l'alto e a sinistra.

2. Agente propenso al rischio (Risk lover). Sia rendimento medio che la volatilità sono beni ed entrambi i coefficienti sono positivi

$$
\begin{gathered}
u(\widetilde{W})=a \mu_{p}+b \sigma_{p}^{2} \\
d U=a d \mu_{p}+\left(2 b \sigma_{p}\right) d \sigma_{p}=0 \\
S M S \equiv \frac{d \mu_{p}}{d \sigma_{p}}=-\frac{2 b \sigma_{p}}{a}<0
\end{gathered}
$$

Disegnamo la curva d'indifferenza $a \mu_{p}+b \sigma_{p}^{2}=\bar{u}$, fissando i parametri $a=4, b=4, \bar{u}=8,16$ (Nota: Per disegnarle, sono costretta ad abbassare l'esponente per mantenere la proprietà di convessità - non preoccupatevene).

$$
\begin{aligned}
a \mu_{p}+b \sigma_{p}^{2} & =\bar{u} \Longrightarrow \mu_{p}=\frac{\bar{u}}{a}-\frac{b \sigma_{p}^{1 / 2}}{a} \\
\mu_{p} & =\frac{8}{4}-\frac{4 x^{1 / 2}}{4} \text { e } \mu_{p}=\frac{16}{4}-\frac{4 x^{1 / 2}}{4}
\end{aligned}
$$

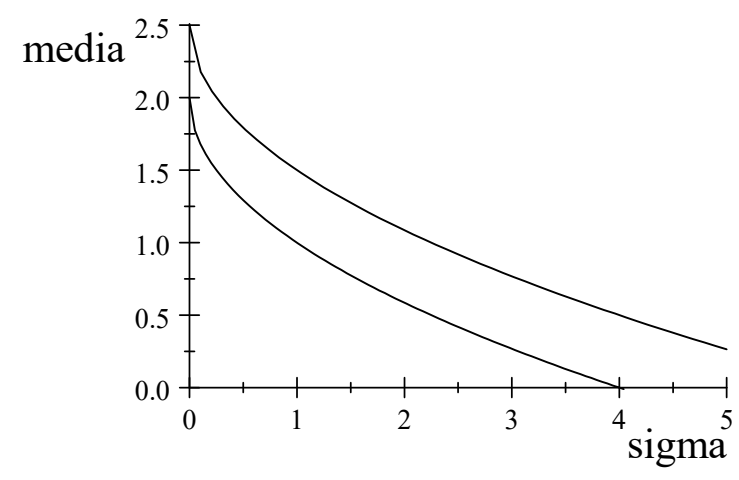

l'utilità cresce nella direzione verso l'alto e a destra (come per i beni normali).

3. Agente neutrale al rischio (Risk neutral). L'investitore ama il rendimento ed è indifferente alla volatilità.

$$
u(\widetilde{W})=a \mu_{p}+0 \sigma_{p}^{2}
$$

Disegnamo la curva d'indifferenza $a \mu_{p}+b \sigma_{p}^{2}=\bar{u}$, fissando i parametri $a=4, b=0, \bar{u}=8,10$.

$$
u(\widetilde{W})=a \mu_{p} \Longrightarrow \mu_{p}=\frac{\bar{u}}{a}=\frac{8}{4} \text { e } \mu_{p}=\frac{\bar{u}}{a}=\frac{10}{4}
$$




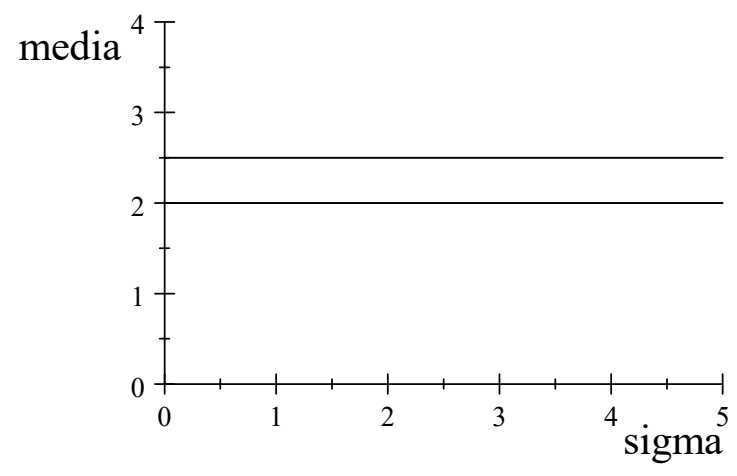

le curve d'indifferenza sono rette orizzontali e l'utilità cresce verso l'alto. Il SMS è costante e nullo.

$$
S M S \equiv \frac{d \mu_{p}}{d \sigma_{p}}=0
$$

\subsection{Oggetti di scelta}

Cosa "sceglie" l'investitore? L'investitore ha a disposizione l'insieme delle attività finanziarie, di cui consideriamo i rendimenti (tassi di varazione dei prezzi di periodicità parametrica, giornalieri, mensili, annuali). Egli decide le quote $w_{k}, k=1, \ldots, K$ in cui detenerle in portafoglio affinché il rendimento medio del portafoglio sia massimo, per varianza data, o la varianza sia minima, per rendimento dato.

Considerando le ipotesi sui rendimenti citate nell'introduzione, ogni rendimento è quindi descrivibile da un punto sul piano $\left(\sigma_{i}, \mu_{i}\right)$ ed anche il portafoglio sarà un punto sul piano $\left(\sigma_{P}, \mu_{P}\right)$ che varia al variare dei pesi $w_{k}$ in cui sono detenute le diverse attività.

Definizione 1 Un "portafoglio" è una combinazione lineare di attività finanziarie $\widetilde{r}_{k}$ mediante un vettore di "pesi" percentuali che sommano ad uno.

$$
\{w\}_{k=1}^{K} \text {, tali che } \sum_{k=1}^{K} w_{k}=1
$$

Essendo un portafoglio una combinazione di variabile aleatorie sarà anche esso una variabile aleatoria di cui defininiamo il rendimento aleatorio

$$
\widetilde{r}_{p}=\sum_{k=1}^{K} w_{k} \widetilde{r}_{k}
$$

In forma matriciale

$$
\widetilde{r}_{p} \sim N\left(\mu_{P}, \sigma_{P}\right)=N\left(\mathbf{w}^{\prime} \boldsymbol{\mu}, \mathbf{w}^{\prime} \mathbf{\Sigma} \mathbf{w}\right)
$$

Dove

$$
\begin{aligned}
& \mu_{P} \stackrel{\text { def }}{=} E\left(\widetilde{r}_{P}\right)=\sum_{k=1}^{K} w_{k} E\left(\widetilde{r}_{k}\right)=\sum_{k=1}^{K} w_{k} \mu_{k}=\mathbf{w}^{\prime} \boldsymbol{\mu} \\
\sigma_{P}^{2} \stackrel{\text { def }}{=} \operatorname{Var}\left(\widetilde{r}_{p}\right)= & \sum_{k=1}^{K} w_{k}^{2} E\left[r_{k}-E\left(\widetilde{r}_{k}\right)\right]^{2}+\sum_{k=1}^{K} \sum_{j \neq k}^{K} w_{k} w_{j} E\left[r_{k}-E\left(\widetilde{r}_{k}\right)\right] E\left[r_{j}-E\left(\widetilde{r}_{j}\right)\right] \\
= & \mathbf{w}^{\prime} \mathbf{\Sigma} \mathbf{w}
\end{aligned}
$$


Definizioni ed esempi per $K=2$.

$$
\begin{aligned}
\mu_{P} \stackrel{\text { def }}{=} E\left(\widetilde{r}_{p}\right) & =\mathbf{w}^{\prime} \boldsymbol{\mu} \\
& =\left(\begin{array}{ll}
w_{1} & w_{2}
\end{array}\right)\left(\begin{array}{c}
\mu_{1} \\
\mu_{2}
\end{array}\right) \\
& =w_{1} \mu_{1}+w_{2} \mu_{2}
\end{aligned}
$$

where $\sigma_{12}=\sigma_{21} \leq \rho \sigma_{1} \sigma_{2}$, poiché $\rho \in[-1,1]$.

Possiamo calcolare il coefficiente $\rho^{*}$ di correlazione tale per cui

$$
\sigma_{12}=\sigma_{21}=\rho^{*} \sigma_{1} \sigma_{2}
$$

Definizione 2 Coefficiente di correlazione

$$
\rho_{i j}^{*}=\frac{\operatorname{cov}\left(\widetilde{r}_{1}, \widetilde{r}_{2}\right)}{\sigma_{i} \sigma_{j}}=\frac{\sigma_{i j}}{\sigma_{i} \sigma_{j}}
$$

Da cui

$$
\sigma_{P}^{2}=w_{1}^{2} \sigma_{1}^{2}+2 w_{1} w_{2} \rho^{*} \sigma_{1} \sigma_{2}+w_{2}^{2} \sigma_{2}^{2}
$$

Definizione 3 "Attività senza rischio". Attività con rendimento costante e dunque varianza nulla

$$
r_{f} \sim\left(r_{f}, 0\right)
$$

\section{$3 \quad$ Insieme dei portafogli possibili}

Il rendimento del portafoglio $\widetilde{r}_{p}$, è, come già detto, una variabile aleatoria. Per poterlo utilizzare nei calcoli viene "fotografato" nella media e nella varianza.

Cerchiamo la frontiera efficiente di tutti i portafogli possibili date le attività finanziarie disponibili. Ovvero cerchiamo come varia il punto $\left(\sigma_{P}, \mu_{P}\right)$ al variare del vettore dei pesi $\mathbf{w}=\left(w_{1}, \ldots, w_{k}, \ldots w_{K}\right)$.

Per fare ciò

1. risolviamo la varianza del portafoglio per i pesi $w_{k}$ in funzione di $\sigma_{P}$ : il vettore $\mathbf{w}\left(\sigma_{P}\right)$

2. sostituiamo poi tali $w_{k}\left(\sigma_{P}\right)$ nell'equazione del valore atteso $\mu_{P}$.

In questo modo otteniamo appunto la "frontiera efficiente di tutti i portafogli possibili date le attività finanziarie disponibili".

Consideriamo nel seguito i casi in cui il portafoglio sia costituito da due sole attività finanziarie

Consideriamo il piano $\left(\sigma_{P}, \mu_{P}\right)$ nel quale ogni punto costituisce un portafoglio. Per poter avere calcoli trattabili consideriamo un portafoglio formato da sole due attività finanziarie e consideriamo la frontiera nei due casi:

- Caso 1 - Un'attività rischiosa e una senza rischio: $\Longrightarrow$ la frontiera efficiente è rappresentata da una retta, la "capital market line" (CML).

- Caso 2 - Due attività rischiose: $\Longrightarrow$ la frontiera efficiente è rappresentata dalla frontiera superiore della curva di isovarianza.

- Caso 3 - Due attività rischiose (l'indice di mercato e un'attività finanziaria che faccia parte dell'indice): $\Longrightarrow$ CAPM. 


\subsection{Caso 1 - Frontiera efficiente con un'attività rischiosa e una senza rischio: "capital market line"}

Introduciamo un'attività senza rischio, ovvero che ha un rendimento dato.

osservazione 1 L'attività senza rischio introduce la possibilità di prendere e dare a prestito. Ovvero introduce la possibilità di scambiare su un mercato. Determina un prezzo di mercato del rischio ed equivale, nel ruolo, al vincolo di bilancio.

Consideramo il rendimento di un portafoglio contenente un'attività rischiosa $r_{i} \sim N\left(\mu_{i}, \sigma_{i}^{2}\right)$, che abbia una distribuzione normale e quindi sia definibile dai soli primi due momentied una attività senza rischio, che definizione vale in media in tasso senza rischio ed ha una varianza nulla $r_{f} \sim\left(r_{f}, 0\right)$. Sia

Definizione $4 w$ = quota del portafoglio detenuta nell'attività a rendimento aleatorio (rischiosa)

Definizione 5 Il rendimento di portafoglio, $\tilde{r_{P}}$ è una variabile aleatoria

$$
\tilde{r_{P}}=w \tilde{r_{i}}+(1-w) \cdot r_{f}
$$

Una variabile aleatoria non è quantificabile. Per poterla inserire nei calcoli dobbiamo trattare i suoi primi due momenti:

- il valor medio del rendimento del portafoglio

$$
\mu_{p}=w \mu_{k}+(1-w) r_{f}
$$

- la varianza del portafoglio

$$
\sigma_{P}^{2}=w^{2} \sigma_{k}^{2}+(1-w)^{2} \cdot 0+2 w(1-w) \cdot 0
$$

- lo scarto quadratico medio del portafoglio

$$
\sigma_{p}=w \sigma_{i}
$$

Dall'equazione (4) ricavare

In questo caso semplice, si può sostituire direttamente la (4) esplicitata per $w$ nella (3) ed ottenere la

Definizione 6 Capital Market Line. La retta che esprime il valore atteso (media) del portafoglio, al crescere del rischio (scarto quadratico medio) al variare della quota $x$ di attività rischiosa

$$
\begin{aligned}
\mu_{p} & =r_{f}+w\left(\mu_{i}-r_{f}\right) \\
& =r_{f}+\left(\frac{\mu_{i}-r_{f}}{\sigma_{i}}\right) \sigma_{p}
\end{aligned}
$$

Mettiamo la (5) in grafico esplicitando per $\mu_{p}$ :

- $r_{f}$ è l'intercetta e rappresenta per l'appunto l'attività finanziaria senza rischio che da un rendimento $r_{f}$ e un rischio $\sigma_{p}=0$. Lo stesso punto esprime un portafoglio investito nella sola attività senza rischio ovvero in cui $w=0$.

Definizione 7 Il coefficiente angolare della retta rappresenta il "premio per il rischio" su questo portafoglio. Ovvero quanto rendimento medio in eccesso sul rendimento senza rischio un'attività deve fornire se ha una volatilità pari a $\sigma_{i}$. 
- Esempio grafico per

$$
\mu_{p}=0.03+\left(\frac{0.1-0.03}{0.2}\right) \sigma_{p}
$$

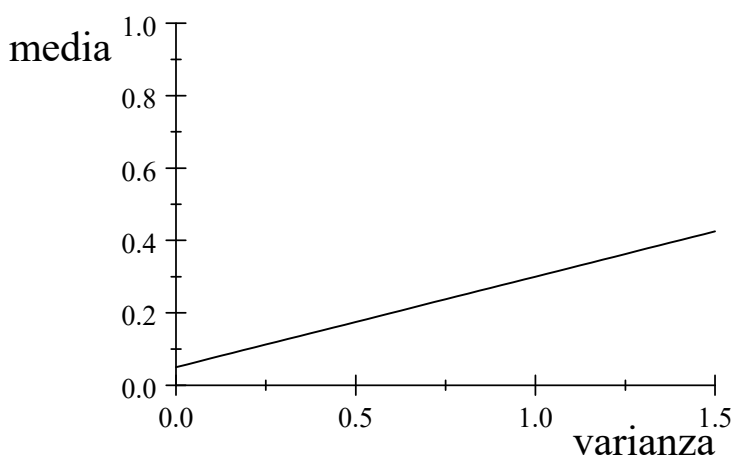

osservazione 2 La Capital Market Line è in sostanza il "vincolo di bilancio" per l'investitore.

- Ogni portafoglio al di sotto di tale retta per un pari rendimento medio offre una rischio(o, sinonimo, volatilità) maggiore, oppure a pari richio offre un rendimento minore. Questa retta sarà l'analogo del vicolo di bilancio nel caso della scelta del consumatore.

\section{Caso 2 - Frontiera efficiente con 2 attività rischiose}

Metodo.

Stiamo cercando il luogo dei punti di isovarianza al variare del vettore dei pesi $\mathbf{w}$.

Esplicitiamo quindi $\sigma_{P}^{2}(w)$, avendo tutti i dati di w e $\boldsymbol{\Sigma}$.

Per semplificare, considerando soltanto due attività rischiose, abbiamo soltanto i pesi $w$ e $(1-w)$ e quindi in sostanza la sola incognita $w$.

$$
\begin{aligned}
\sigma_{P}^{2} & =\left(\begin{array}{ll}
w & 1-w
\end{array}\right)\left(\begin{array}{cc}
0.15 & 0.0725 \\
0.0725 & 0.27
\end{array}\right)\left(\begin{array}{c}
w \\
1-w
\end{array}\right) \\
& =0.275 w^{2}-0.395 w+0.27
\end{aligned}
$$

Risulta che $\sigma_{P}^{2}$ è una equazione di II grado in $w$.

Invertire la funzione risolvendo per $w\left(\sigma_{P}^{2}\right)$

$$
w\left(\sigma_{P}^{2}\right)= \begin{cases}0.71818+1.8182 \sqrt{1.1 \sigma_{P}^{2}-0.14098}, & \text { Soluzione } 1 \\ 0.71818-1.8182 \sqrt{1.1 \sigma_{P}^{2}-0.14098}, & \text { Soluzione } 2\end{cases}
$$

L'equazione è una parabola di II grado, quindi vi sono due soluzioni.

Inserire ognuna delle soluzioni nel vincolo del rendimento atteso.

$$
\mu_{P}=\left(\begin{array}{ll}
w & 1-w
\end{array}\right)\left(\begin{array}{l}
0.34 \\
0.47
\end{array}\right)
$$

Otteniamo quindi due funzioni di rendimento medio $\mu_{P}=f\left(w\left(\sigma_{P}^{2}\right)\right)$ che sono esattamente quelle che voglio disegnare.

Soluzione 1 (nera sottile) 


$$
\begin{aligned}
& \mu_{P}\left(\sigma_{P}^{2}\right)_{1}=\left(1.8182 \sqrt{1.1 \sigma_{P}^{2}-0.14098}+0.718181-\left(1.8182 \sqrt{1.1 \sigma_{P}^{2}-0.14098}+0.71818\right)\right)\left(\begin{array}{l}
0.34 \\
0.47
\end{array}\right) \\
& \mu_{P}\left(\sigma_{P}^{2}\right)=0.37664-0.23637 \sqrt{1.1 \sigma_{P}^{2}-0.14098}
\end{aligned}
$$

La inserisco nel grafico.

Soluzione 2 (ramo verde in grassetto) - Soluzione dominante

$$
\mu_{P}\left(\sigma_{P}^{2}\right)_{2}=\left(0.71818-1.8182 \sqrt{1.1 \sigma_{P}^{2}-0.14098} 1-\left(0.71818-1.8182 \sqrt{1.1 \sigma_{P}^{2}-0.14098}\right)\right)\left(\begin{array}{l}
0.34 \\
0.47
\end{array}\right)=
$$

da cui :

$$
\mu_{P}\left(\sigma_{P}^{2}\right)_{2}=0.23637 \sqrt{1.1 \sigma_{P}^{2}-0.14098}+0.37664
$$

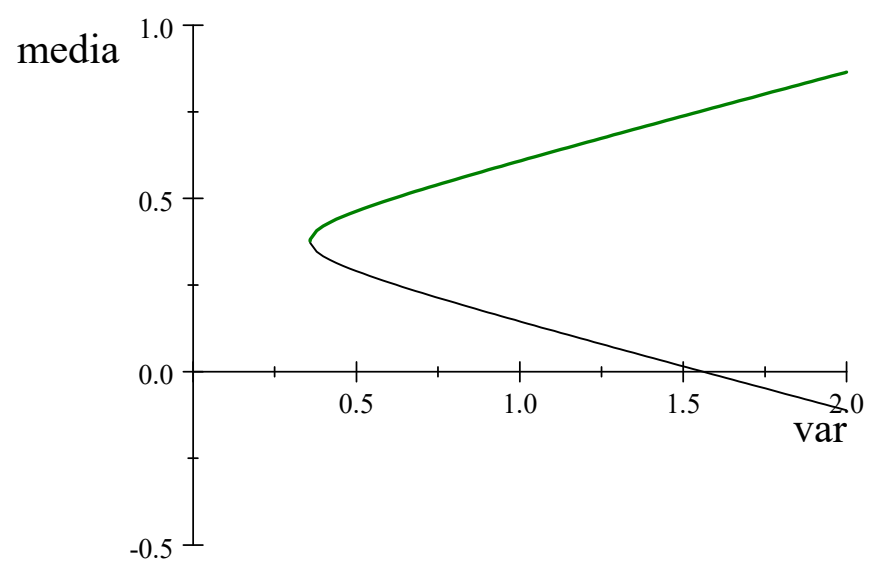

E' chiaro che solo la soluzione più "alta" (in grassetto) è quella dominante, equazione (6), perché à parità di varianza offre il rendimento medio più alto.

Utilizzando quindi la sola soluzione dominante, per ogni $\mu_{P}$ fissato posso trovare la varianza $\sigma_{P}^{2}$ corrispondente, che è minima perché appartiene al ramo dominante, oppure, fissata la varianza trovo il rendimento atteso dominante $\mu_{P}\left(\sigma_{P}^{2}\right)$.

osservazione $3 \mathrm{Nel}$ caso a due dimensioni, è immediatamente visibile quale sia il portafoglio di minima varianza (misurato sell'asse delle ascisse), dato il valore del rendimento medio $\mu_{P}$ (misurato sull'asse delle ordinate) sull'asse delle ordinate. A più dimensioni è necessario risolvere il problema seguente, che svolgiamo per lo stesso caso $K=2$ e $K$ generico.

\subsection{Derivazione portafoglio di minima varianza per rendimento medio dato}

Risolviamo il problema

$$
\begin{aligned}
\min _{\mathbf{w}, \lambda, \gamma} \operatorname{Var}\left(\widetilde{r}_{P}\right) & =\mathbf{w}^{\prime} \mathbf{\Sigma} \mathbf{w} \\
\text { s.t. } \quad \mathbf{w}^{\prime} \boldsymbol{\mu} & \geq \mu_{\text {dato }} \\
\mathbf{w}^{\prime} \mathbf{1}=\mathbf{1} &
\end{aligned}
$$

Deriviamo il lagrangiano (19) rispetto alle incognite $w$ e $\lambda$. Oppure, analogamente, sfruttiamo le derivate di cui sopra in forma matriciale.

$$
\begin{aligned}
\frac{\partial L}{\partial w} & :\left(\begin{array}{ll}
2 & -2
\end{array}\right)\left(\begin{array}{cc}
0.15 & 0.0725 \\
0.0725 & 0.27
\end{array}\right)\left(\begin{array}{c}
w \\
1-w
\end{array}\right)-\lambda\left(\begin{array}{ll}
1 & -1
\end{array}\right)\left(\begin{array}{l}
0.34 \\
0.47
\end{array}\right)=0 \\
& : \quad 0.55 w+0.13 \lambda-0.395=0
\end{aligned}
$$




$$
\begin{gathered}
\frac{\partial L}{\partial \lambda} \quad: \quad \mu_{1} w_{1}+\mu_{2} w_{2}=\mu_{\text {dato }} \\
: \quad 0.47-0.13 w=0.2
\end{gathered}
$$

Dal sistema delle due CPO nelle due incognite, troviamo

$$
\begin{array}{r}
0.55 w+0.13 \lambda-0.395=0 \\
0.47-0.13 w-1.5=0
\end{array}
$$

Soluzioni (in cui lascio $\mu_{\text {dato }}$ )

$$
\left[\begin{array}{c}
w^{*}=3.6154-7.6923 \cdot \mu_{\text {dato }} \\
\lambda^{*}=15.302-35.503 \cdot \mu_{\text {dato }}
\end{array}\right]
$$

Tabella. Risultati per diversi valori di $\mu_{\text {dato }}$

\begin{tabular}{|l|l|l|l|l|l|}
\hline$\mu_{\text {dato }}$ & $20 \%$ & $40 \%$ & $70 \%$ & $100 \%$ & $150 \%$ \\
\hline$w^{*}$ & 2.08 & .54 & -1.77 & -4.077 & -7.92 \\
\hline$\lambda^{*}$ & -5.7 & 0.76 & 10.5 & 20.28 & -36.6 \\
\hline
\end{tabular}




\subsection{Disegno Frontiera efficiente con $K>2$ attività rischiose (Non per l'esame)}

Espongo qui il vero calcolo, solo per gli interessati.

$$
\begin{aligned}
& \min _{\mathbf{w}, \lambda, \gamma} \operatorname{Var}\left(r_{P}\right)=\frac{1}{2} \mathbf{w}^{\prime} \mathbf{\Sigma} \mathbf{w} \\
& \text { s.t. } \quad E\left(r_{P}\right)=\mathbf{w}^{\prime} \boldsymbol{\mu} \geq \mu_{\text {dato }} \\
& \mathbf{w}^{\prime} \mathbf{1}=1 \\
& \min _{w, \lambda} L=\mathbf{w}^{\prime} \mathbf{\Sigma} \mathbf{w}-\lambda\left(\mathbf{w}^{\prime} \boldsymbol{\mu}-r_{f}\right)-\gamma\left(\mathbf{w}^{\prime} \mathbf{1}-1\right) \\
& \frac{\partial L}{\partial \mathbf{w}} \quad: \quad \boldsymbol{\Sigma} \mathbf{w}-\lambda \boldsymbol{\mu}-\gamma \mathbf{1}=0 \\
& \frac{\partial L}{\partial \lambda} \quad: \quad \mathbf{w}^{\prime} \boldsymbol{\mu}-\mu_{\text {dato }}=0 \\
& \frac{\partial L}{\partial \gamma} \quad: \quad \mathbf{w}^{\prime} \mathbf{1}-1=0
\end{aligned}
$$

Reiarangiando i termini della CPO 1

$$
\begin{aligned}
\boldsymbol{\Sigma} \mathbf{w} & =\lambda \boldsymbol{\mu}+\gamma \mathbf{1} \\
\mathbf{w} & =\lambda \boldsymbol{\Sigma}^{-1} \boldsymbol{\mu}+\gamma \boldsymbol{\Sigma}^{-1} \mathbf{1}
\end{aligned}
$$

otteniamo le quote di portafoglio, ma ancora in funzione di $\lambda, \gamma$

$$
\mathbf{w}^{*}=\lambda \boldsymbol{\Sigma}^{-1} \boldsymbol{\mu}+\gamma \boldsymbol{\Sigma}^{-1} \mathbf{1}
$$

Quindi per trovare $\lambda, \gamma$, pre-moltiplichiamo per $\boldsymbol{\mu}^{\prime}$

$$
\boldsymbol{\mu}^{\prime} \mathbf{w}=\lambda \underbrace{\left(\boldsymbol{\mu}^{\prime} \boldsymbol{\Sigma}^{-1} \boldsymbol{\mu}\right)}_{\mathbf{B}}+\gamma \underbrace{\left(\boldsymbol{\mu}^{\prime} \boldsymbol{\Sigma}^{-1} \mathbf{1}\right)}_{\mathbf{A}}=\mathbf{w}^{\prime} \boldsymbol{\mu}=r_{\text {given }}
$$

Premoltiplicando la stessa per il vottore riga e $\mathbf{1}^{\prime}$ invece di $\boldsymbol{\mu}^{\prime}$, otteniamo:

$$
\mathbf{1}^{\prime} \mathbf{w}=\lambda \underbrace{\left(\mathbf{1}^{\prime} \boldsymbol{\Sigma}^{-1} \boldsymbol{\mu}\right)}_{A}+\gamma \underbrace{\left(\mathbf{1}^{\prime} \boldsymbol{\Sigma}^{-1} \mathbf{1}\right)}_{C}=1
$$

Quindi per risolvere per $\lambda$ e $\gamma$, abbiamo il seguente sistema di 2 equazioni in 2 incognite

$$
\begin{gathered}
\left\{\begin{array}{c}
\lambda B+\gamma A=r_{\text {given }} \\
\lambda A+\gamma C=1
\end{array}\right. \\
\lambda^{*}=\frac{C \cdot r_{\text {given }}-A}{B C-A^{2}} \\
\gamma^{*}=\frac{B-A \cdot \mu_{\text {dato }}}{B C-A^{2}}
\end{gathered}
$$

e infine

$$
\begin{aligned}
\mathbf{w}^{*} & =\lambda \boldsymbol{\Sigma}^{-1} \boldsymbol{\mu}+\gamma \boldsymbol{\Sigma}^{-1} \mathbf{1} \\
& =\frac{C r-A}{D} \boldsymbol{\Sigma}^{-1} \boldsymbol{\mu}+\frac{B-A r}{D} \boldsymbol{\Sigma}^{-1} \mathbf{1} \\
& =\underbrace{\left[\frac{B\left(\boldsymbol{\Sigma}^{-1} \mathbf{1}\right)-A\left(\boldsymbol{\Sigma}^{-1} \boldsymbol{\mu}\right)}{D}\right]}_{g}+\underbrace{\left[\frac{C\left(\boldsymbol{\Sigma}^{-1} \boldsymbol{\mu}\right)-A\left(\boldsymbol{\Sigma}^{-1} \mathbf{1}\right)}{D}\right]}_{h} \cdot \mu_{\text {dato }} \\
& =\text { constant }+h \cdot \mu_{\text {dato }}
\end{aligned}
$$


Se $\mu_{\text {dato }}=0 \Longrightarrow \mathbf{w}^{*}=g$

If $\mu_{\text {dato }}=1 \Longrightarrow \mathbf{w}^{*}=g+h$

Quindi il valore calcolato di

$$
\begin{aligned}
\mu_{P} & =\mathbf{w}^{* \prime} \cdot \boldsymbol{\mu} \\
\sigma_{P}^{2} & =\mathbf{w}^{* \prime} \cdot \mathbf{\Sigma} \cdot \mathbf{w}^{*} \\
& =\left(g+h \cdot r_{\text {given }}\right)^{\prime} \cdot \mathbf{\Sigma} \cdot\left(g+h \cdot r_{\text {given }}\right) \\
& =\cdots \\
& =\frac{1}{C}+\frac{C}{D}\left[r_{\text {given }}-\frac{A}{C}\right]^{2}
\end{aligned}
$$

Ma noi abbiamo bisogno della funzione

\subsubsection{Disegno sul piano $(\sigma, \mu)$}

Risolvendo quest'ultima espressione per $E\left(r_{P}\right)$

$$
\mu_{P}=\frac{A}{C} \pm \sqrt{\frac{D}{C}\left(\operatorname{Var}\left(r_{P}\right)-\frac{1}{C}\right)}
$$

Grazie ai calcoli matriciali in Matlab

$$
\mu_{P}=0.353 \pm \sqrt{124.46(x-0.0053)}
$$

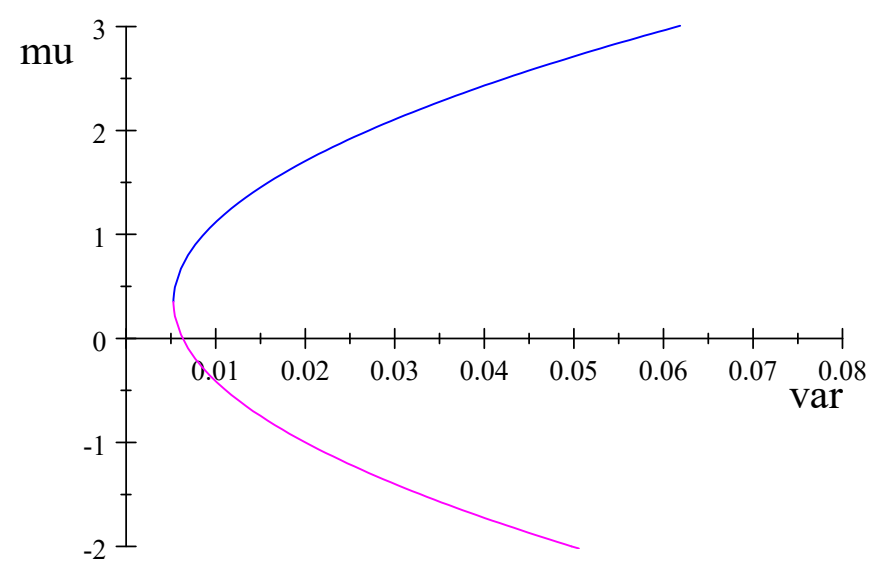

dove

- il portfolio di varianza minima (vertice della parabola) ha coordinate $\left(\frac{1}{C}, \frac{A}{C}\right)=(0.0053,0.353)$;

\subsubsection{Disegno sul piano $(\sigma, \mu)$}

$$
\sigma\left(\widetilde{r_{P}}\right)=\sqrt{\frac{1}{C}+\frac{C}{D}\left[E\left(r_{P}\right)-\frac{A}{C}\right]^{2}}
$$

Solving for $E\left(r_{P}\right)$

$$
E\left(r_{P}\right)=\frac{1}{C}\left(A \pm D \sqrt{\frac{1}{D}\left(C x^{2}-1\right)}\right)
$$


Using matlab Computations

$$
E\left(r_{P}\right)=0.0053\left(6.623 \pm 233570000 \sqrt{\frac{1}{233570000}\left(187.66 \cdot \sigma^{2}\left(\widetilde{r_{P}}\right)-1\right)}\right)
$$

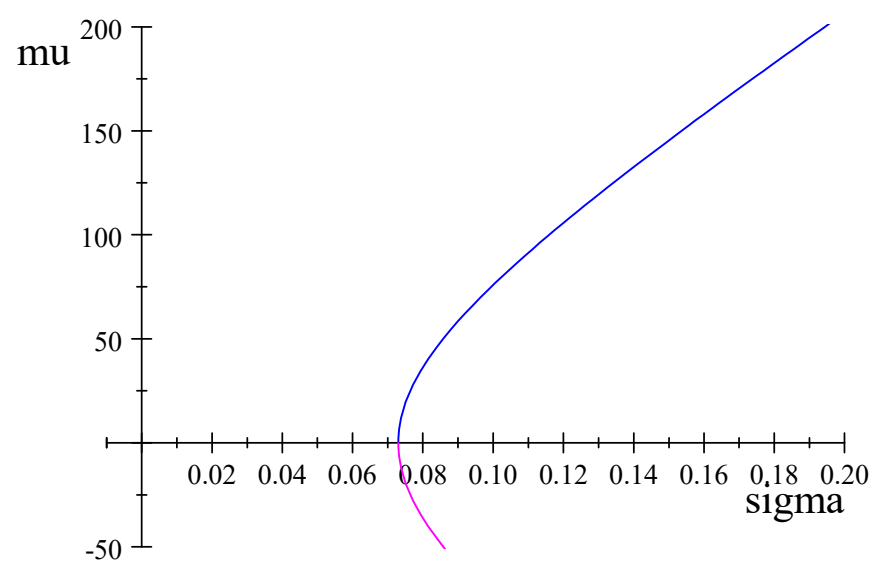

Zooming in

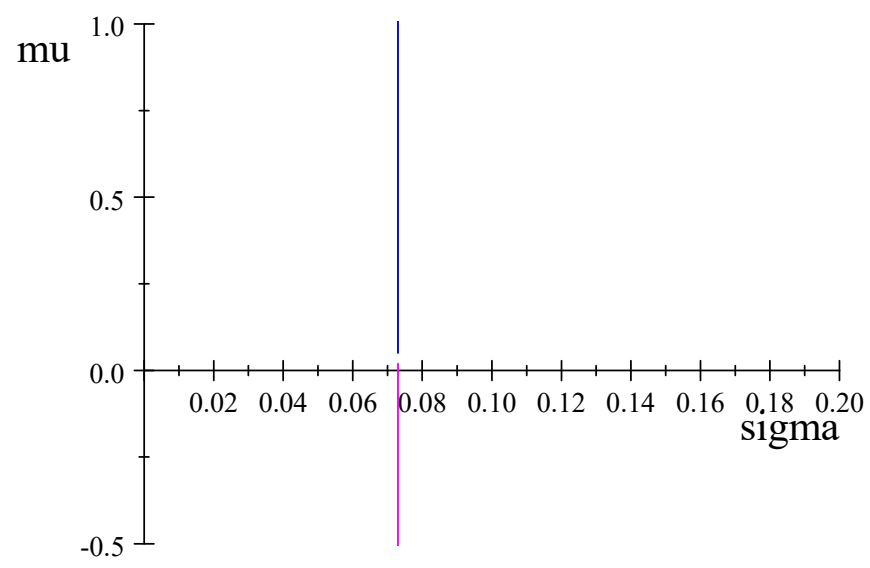

Where

- the min sigma portfolio (vertex of parabola) has coordinates $\left(\sqrt{\frac{1}{C}}, \frac{A}{C}\right)=(0.0730,0.353)$

\section{Scelta ottima (SI)}

Dopo aver definito la funzione di scelta e la frontiera efficiente dobbiamo mettere le due cose insieme e definire la scelta ottima.

Consideriamo i Casi 1 e 2 menzionati.

\subsection{Scelta Ottima: portafoglio con un'attività risk-free e un'attività rischiosa}

Dati

$$
\boldsymbol{\mu}=\left(\begin{array}{c}
\mu_{i} \\
r_{f}
\end{array}\right), \quad \boldsymbol{\Sigma}=\left(\begin{array}{cc}
\sigma_{1}^{2} & 0 \\
0 & 0
\end{array}\right)
$$


$a>0$ e $b>0$, in una funzione di utilità che vede la varianza come un "male". Ottimizzazione di portafoglio con un'attività rischiosa ed una risk-free detenuti rispettivamente nelle quote $w$ e $(1-w)$

$$
\begin{aligned}
\max _{\sigma_{P}, \mu_{P}} u\left(\sigma_{P}^{2}, \mu_{P}\right) & =a \mu_{P}-b \sigma_{P}^{2} \\
\text { t.c. } \quad \tilde{r_{p}} & =w \tilde{r}_{i}+(1-w) r_{f}
\end{aligned}
$$

La variabile aleatoria del portafoglio va tradotta in termini di media e varianza del portafoglio

$$
\begin{aligned}
& \mu_{p}=w \mu_{i}+(1-w) r_{f} \\
& \sigma_{p}=w \sigma_{i}+(1-w) \cdot 0
\end{aligned}
$$

A questo punto si può sia trattare il problema come un problema di ottimo con due vincoli

$$
\begin{gathered}
\max u\left(\sigma_{p}^{2}, \mu_{p}\right)_{\sigma_{p}^{2}, \mu_{p}}=a \mu_{p}-b \sigma_{p}^{2} \\
\text { t.c. } \quad\left\{\begin{array}{l}
\mu_{p}=w \cdot \mu_{i}+(1-w) r_{f} \\
\sigma_{p}=w \cdot \sigma_{i}
\end{array}\right.
\end{gathered}
$$

oppure si sostituisce il secondo vincolo nel primo per mezzo di $w$ (possibile solo nel caso di un'attività rischiosa e una senza rischio) e si ottiene la CML, ovvero la frontiera efficiente.

$$
\begin{gathered}
\max u\left(\sigma_{p}^{2}, \mu_{p}\right)_{\sigma_{p}, \mu_{p}}=a \mu_{p}-b \sigma_{p}^{2} \\
\text { t.c. } \quad \mu_{p}=r_{f}+\left(\frac{\mu_{i}-r_{f}}{\sigma_{i}}\right) \sigma_{p} \\
L=a \mu_{p}-b \sigma_{p}^{2}-\lambda\left[\mu_{p}-r_{f}-\left(\frac{\mu_{i}-r_{f}}{\sigma_{i}}\right) \sigma_{p}\right] \\
\frac{\partial L}{\partial \sigma_{P}} \quad: \quad-2 b \sigma_{p}+\lambda\left(\frac{\mu_{i}-r_{f}}{\sigma_{i}}\right)=0 \\
\frac{\partial L}{\partial \mu_{P}} \quad: \quad a-\lambda=0 \\
\frac{\partial L}{\partial \lambda} \quad: \quad \mu_{p}-r_{f}-\left(\frac{\mu_{i}-r_{f}}{\sigma_{i}}\right) \sigma_{p}=0
\end{gathered}
$$

Dalla $(10) \lambda^{*}=a$. Dividendo la $(1) /(2)$

$$
\begin{aligned}
\frac{2 b \sigma_{p}}{a} & =\frac{\mu_{i}-r_{f}}{\sigma_{i}} \\
S M S & =\text { prezzo del rischio }
\end{aligned}
$$

si ricava

$$
\sigma_{p}^{*}=\frac{\mu_{i}-r_{f}}{\sigma_{i}} \frac{a}{2 b}
$$

sostituendo il valore trovato nella (3) si trova

$$
\mu_{p}^{*}=r_{f}+\left(\frac{\mu_{i}-r_{f}}{\sigma_{i}}\right) \sigma_{p}^{*}
$$

Per trovare $w^{*}$ si usa la (8)

$$
w^{*}=\sigma_{p}^{*} / \sigma_{i}
$$




\subsubsection{Esempio numerico}

Dati $r_{i} \sim\left(\mu_{i}, \sigma_{i}^{2}\right)=\left(0.15,0.2^{2}\right), \quad r_{f} \sim\left(r_{f}, 0\right)=(0.05,0), \quad a=10, b=5$.

Soluzione. Applicare i parametri allo svolgimento dell'esercizio, qui sopra. Si ha

$$
\begin{aligned}
\lambda^{*} & =10 \\
\sigma_{p}^{*} & =\frac{\mu_{i}-r_{f}}{\sigma_{i}} \frac{a}{2 b}=\frac{0.15-0.05}{0.2} \frac{10}{2 \cdot 5}=0.5 \\
\mu_{p}^{*} & =r_{f}+\left(\frac{\mu_{i}-r_{f}}{\sigma_{i}}\right) \sigma_{p}^{*}=0.05+\left(\frac{0.15-0.05}{0.2}\right) 0.5=0.3 \\
w^{*} & =\sigma_{p}^{*} / \sigma_{i}=0.5 / 0.2=2.5 \\
u^{*}\left(\mu_{p}^{*}, \sigma_{p}^{* 2}\right) & =a \mu_{p}^{*}-b \sigma_{p}^{* 2}=10 \cdot 0.3-5 \cdot 0.5^{2}=1.75
\end{aligned}
$$

Per mettere in grafico la curva d'indifferenza tangente, si esprile l'utilità risolvendo per $\mu_{P}$

$$
\mu_{p}=\frac{u^{*}}{a}+\frac{b}{a} \sigma_{p}^{* 2}=\frac{1.75}{10}+\frac{5}{10} \sigma_{p}^{2}
$$

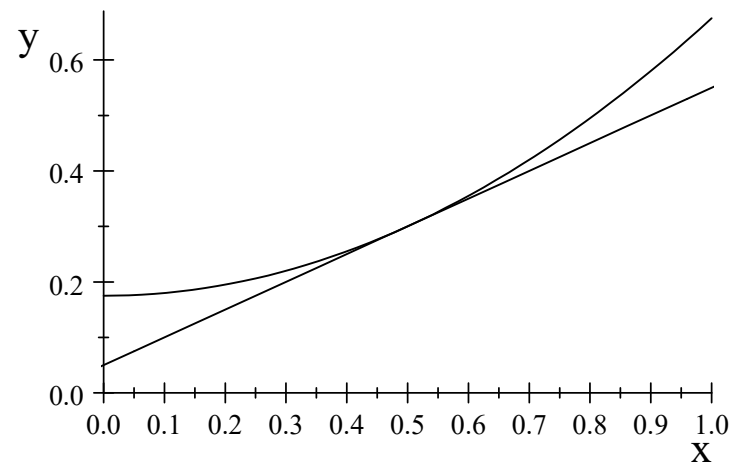

Il fatto che la quota di portafoglio $w^{*}=2.5$ significa che la scelta ottima è acquistare l'attività senza rischio $\left(=\right.$ indebitarsi) per il $\left(1-w^{*}\right)=1-2.5=-150 \%$ ovvero indebitarsi per il $150 \%$ della ricchezza e investire il $250 \%$ nell'attività rischiosa.

Se localizzate l'attività $r_{i} \sim\left(\mu_{i}, \sigma_{i}^{2}\right)$ sul piano, e sapere che quel punto corrisponde ad una quota del $100 \%$, vi accorgete che la scelta ottima è due volte e mezzo più in avanti sulla curva.

\subsection{Scelta Ottima: portafoglio con due attività rischiose}

Abbiamo il problema di cercare i pesi per un portafoglio che dia un rendimento massimo, dove le attività a disposizione sono due attività rischiose distribuite con il seguente vettore delle medie e matrice di covarianza

$$
\begin{aligned}
\max _{\sigma_{P}, \mu_{P}} u\left(\sigma_{P}^{2}, \mu_{P}\right) & =a \mu_{P}-b \sigma_{P}^{2} \\
\text { t.c. } \quad \tilde{r}_{p} & =w \tilde{r}_{i}+(1-w) r_{f}
\end{aligned}
$$

dove

$$
\widetilde{r_{P}} \sim N\left(\mathbf{w}^{\prime} \boldsymbol{\mu}, \mathbf{w}^{\prime} \mathbf{\Sigma} \mathbf{w}\right)
$$

e dove

$$
\boldsymbol{\mu}=\left(\begin{array}{l}
\mu_{1} \\
\mu_{2}
\end{array}\right), \quad \boldsymbol{\Sigma}=\left(\begin{array}{ll}
\sigma_{1}^{2} & \rho_{12} \sigma_{1} \sigma_{2} \\
\rho_{21} \sigma_{1} \sigma_{2} & \sigma_{2}^{2}
\end{array}\right)
$$


La variabile aleatoria del portafoglio va tradotta in termini di media e varianza del portafoglio

$$
\left\{\begin{array}{l}
\mu_{P}=\mathbf{w}^{\prime} \boldsymbol{\mu} \\
\sigma_{P}=\mathbf{w}^{\prime} \Sigma \mathbf{w}
\end{array}\right.
$$

ovvero

$$
\begin{gathered}
\mu_{P}=\mathbf{w}^{\prime} \boldsymbol{\mu} \\
=\left(\begin{array}{ll}
w & 1-w
\end{array}\right)\left(\begin{array}{c}
\mu_{1} \\
\mu_{2}
\end{array}\right) \\
\mu_{P}=w \mu_{1}+(1-w) \mu_{2} \\
\sigma_{P}^{2}=\mathbf{w}^{\prime} \mathbf{\Sigma} \\
=\left(\begin{array}{cc}
w & 1-w
\end{array}\right)\left(\begin{array}{cc}
\sigma_{1}^{2} & \rho_{12} \sigma_{1} \sigma_{2} \\
\rho_{12} \sigma_{1} \sigma_{2} & \sigma_{2}^{2}
\end{array}\right)\left(\begin{array}{c}
w \\
1-w
\end{array}\right) \\
\sigma_{P}^{2}=w^{2} \sigma_{1}^{2}+(1-w)^{2} \sigma_{2}^{2}+2 w(1-w) \rho_{12} \sigma_{1} \sigma_{2}
\end{gathered}
$$

Metodo:

(i) Risolviamo $\sigma_{P}^{2}$ per $w$. Si tratta di un'equazione di II grado, come già spiegato nel Par. 4.

(ii) Inseririamo ognuna delle due $w\left(\sigma_{P}^{2}\right)$ in (13) ottenendo un' equazione $\mu_{P}\left(w\left(\sigma_{P}^{2}\right)\right)$ per ogni soluzione. Sono i grafici dei due rami di parabola. Trascuriamo il ramo dominato (più basso) e quello più alto diventa il vincolo dell'ottimizzazione dell'utilità, che chiameremo $\mu_{P}^{*}\left(w\left(\sigma_{P}^{2}\right)\right)$.

Il problema di ottimo diventa

$$
\begin{aligned}
\max _{\sigma_{P}, \mu_{P}} u\left(\sigma_{P}^{2}, \mu_{P}\right)= & a \mu_{P}-b \sigma_{P}^{2} \\
& \text { t.c. } \quad \mu_{P}^{*}\left(w\left(\sigma_{P}^{2}\right)\right)
\end{aligned}
$$

La soluzione può essere svolta soltanto numericamente.

\section{CAPM (Capital Asset Pricing Model)}

Definizione 8 Il modello CAPM definisce i rendimenti d'equilibrio delle attività rischiose in funzione della covarianza con il portafoglio di mercato

$$
E\left(\tilde{r_{k}}\right)=r_{f}+\frac{\sigma_{i m}}{\sigma_{m}^{2}}\left[E\left(\tilde{r_{M}}\right)-r_{f}\right]
$$

dove

$$
\beta_{i} \stackrel{\text { def }}{=} \frac{\sigma_{i m}}{\sigma_{m}^{2}}
$$

Nel seguito dimostreremo come e sotto quali ipotesi viene derivata questa formula.

Obiettivi del CAPM:

1. determinare il prezzo di mercato del rischio;

2. determinare la misura appropriata del rischio per la singola attività.

Definizione 9 "Portafoglio di mercato" $R_{m}$. Portafoglio contenente tutte le attività scambiabili sul mercato tenute in proporzione al loro valore relativo ai prezzi d'equilibrio

$$
w_{i}=\frac{\text { valore di mercato dell'att. singola }}{\text { valore di mercato di tutte le att. }}
$$

Es. Indice MIB per la borsa di Milano. 
Definizione 10 "Equilibrio di mercato". I prezzi di tutte le attività sono tali per cui la domanda eguagli l'offerta per tutte le attività.

Ipotesi 1 Tutti gli agenti hanno aspettative omogenee sui rendimenti. In particolare, in questo modello, gli agenti condividono una funzione di densità congiunta Normale (o Gaussiana) sulla totalità dei rendimenti.

Questa fortissima ipotesi implica la seguente identità

Ipotesi 2 Il portafoglio di mercato e' definito come il portafoglio di tangenza fra l'insieme dei portafogli rischiosi efficienti e la "capital market line".

$$
M \stackrel{\text { def }}{\equiv} T
$$

La dimostrazione si basa sul fatto che, dal momento che gli investitori hanno aspettative omogenee, essi percepiranno lo stesso insieme di opportunità di minima varianza.

Non appena le aspettative varierano, i rendimenti attesi e le varianze dei titoli saranno percepite come diverse. Pertanto $M \neq T^{i}$ dove quest'ultimo rappresenta il portafoglio di tangenza per l'investitore $i-$ esimo.

\subsection{Derivazione del CAPM}

Consideriamo un portafoglio cha abbia una quota $a$ investita nell'attività rischiosa $i$ - esima ed una quota $(1-a)$ nel portafoglio di mercato $m$. Il rendimento atteso del portafoglio sarà

$$
\begin{aligned}
& E\left(\widetilde{r}_{C A P M}\right)=\alpha \cdot E\left(\widetilde{r}_{k}\right)+(1-\alpha) E\left(\widetilde{r}_{M}\right) \\
& \operatorname{Var}\left(\widetilde{r}_{C A P M}\right)=\left(\begin{array}{ll}
a & (1-a)
\end{array}\right)\left(\begin{array}{cc}
\sigma_{k}^{2} & \sigma_{k M} \\
\sigma_{k M} & \sigma_{M}^{2}
\end{array}\right)\left(\begin{array}{c}
a \\
1-a
\end{array}\right) \\
& =a^{2} \sigma_{M}^{2}+a^{2} \sigma_{k}^{2}-2 \sigma_{M k} a^{2}-2 a \sigma_{M}^{2}+2 \sigma_{M k} a+\sigma_{M}^{2} \\
& \sigma\left(\widetilde{r}_{C A P M}\right)=\sqrt{a^{2} \sigma_{M}^{2}+a^{2} \sigma_{k}^{2}-2 \sigma_{k, M} a^{2}-2 a \sigma_{M}^{2}+2 \sigma_{k, M} a+\sigma_{M}^{2}} \\
& \sigma\left(\tilde{r_{P}}\right)=\sqrt{a^{2} \sigma_{k}^{2}+(1-a)^{2} \sigma_{M}^{2}+2 a(1-a) \sigma_{k, M}}
\end{aligned}
$$

dove $\sigma_{k, M}$ è un altro modo di scrivere $\operatorname{cov}(k, M)$.

$$
\frac{\partial \sigma\left(\widetilde{r}_{C A P M}\right)}{\partial a} \mid=\frac{2 \sigma_{k, M}-4 a \sigma_{k, M}-2 \sigma_{M}^{2}+2 a \sigma_{k}^{2}+2 a \sigma_{M}^{2}}{2 \sqrt{a^{2} \sigma_{k}^{2}+a^{2} \sigma_{M}^{2}+2 a \sigma_{k, M}+\sigma_{M}^{2}-2 a \sigma_{M}^{2}-2 a^{2} \sigma_{k, M}}}
$$

osservazione 4 L'attività $i$ - esima appartiene al portafoglio di mercato in quota pari a $\left(w_{i}+a\right)$, dunque a rappresenta l'eccesso di domanda dell'attività $i$-esima. Pertanto in equilibrio deve essere $a=0$.

Dunque in equilibrio bisogna calcolare le due derivate nel punto $a=0$.

$$
\begin{aligned}
\left.\frac{\partial E\left(\widetilde{r}_{C A P M}\right)}{\partial a}\right|_{a=0} & =E\left(\widetilde{r}_{k}\right)-\mu_{M} \\
\left.\frac{\partial \sigma\left(\widetilde{r}_{C A P M}\right)}{\partial a}\right|_{a=0} & =\frac{2 \sigma_{k, M}-2 \sigma_{M}^{2}}{2 \sqrt{\sigma_{M}^{2}}} \\
& =\frac{\sigma_{k, M}-\sigma_{M}^{2}}{\sigma_{M}} \\
& =\frac{0.04-0.05}{\sqrt{0.05}}
\end{aligned}
$$


osservazione 5 Affinché il CAPM "funzioni" è necessario che sia $\sigma_{k, M}>\sigma_{M}^{2}$.

$$
\begin{aligned}
\left.\frac{\partial E\left(\widetilde{r}_{C A P M}\right)}{\partial \sigma\left(\widetilde{r}_{C A P M}\right)}\right|_{a=0} & =\frac{\left.\frac{\partial E\left(\widetilde{r}_{C A P M}\right)}{\partial a}\right|_{a=0}}{\left.\frac{\partial \sigma\left(\widetilde{r}_{C A P M}\right)}{\partial a}\right|_{a=0}} \\
& =\frac{\mu_{k}-\mu_{M}}{\frac{\sigma_{k, M}-\sigma_{M}^{2}}{\sigma_{M}}}
\end{aligned}
$$

Cerchiamo il punto della frontiera efficiente dell'insieme dei portafogli con attività rischiose che sia tangente alla CML dell'investitore.

Dobbiamo quindi eguagliare la pendenza dell'insieme dei portafogli con attività rischiose nel punto del portafoglio di mercato e la pendenza della CML (che rappresenta "il prezzo relativo di mercato") (che si trova considerando un'attività rischiosa e una senza rischio).

Tale condizione di tangenza definisce il "beta" ovvero la correlazione fra la proporzione del rischio di mercato contenuta nell'attività $k$-esima e ed il rendimento atteso che deve fornire per essere inclusa nell'indice di mercato (ovvero essere desiderabile) per l'investitore.

In equilibrio la pendenza in $M$ deve essere pari alla pendenza della "capital market line" che rappresenta "il prezzo relativo di mercato", la quale era $\left[\mu_{M}-r_{f}\right] / \sigma_{M}$.

$$
\begin{aligned}
\left.\frac{\partial E\left(\widetilde{r}_{C A P M}\right)}{\partial \sigma\left(\widetilde{r}_{C A P M}\right)}\right|_{a=0} & =\frac{\mu_{M}-r_{f}}{\sigma_{M}} \\
\frac{E\left(\widetilde{r}_{k}\right)-\mu_{M}}{\frac{\sigma_{k, M}-\sigma_{M}^{2}}{\sigma_{M}}} & =\frac{\mu_{M}-r_{f}}{\sigma_{M}}
\end{aligned}
$$

Risolvendo per $\mu_{k}$

$$
\begin{aligned}
& E\left(\widetilde{r}_{k}\right) \stackrel{\operatorname{defCAPM}}{=} r_{f}+\frac{\sigma_{k, M}}{\sigma_{M}^{2}}\left(\mu_{M}-r_{f}\right) \\
= & r_{f}+\beta_{k M}\left(\mu_{M}-r_{f}\right)
\end{aligned}
$$

Ponendo la pendenza dell'insieme nel punto di equilibrio di mercato (M) (??) pari alla pendenza della "capital market line" rispetto al portafoglio di tangenza (??)

\section{Soluzioni numeriche}

$$
\begin{gathered}
\boldsymbol{\mu}=\left(\begin{array}{c}
\mu_{k}=0.20 \\
\mu_{M}=0.10
\end{array}\right), \quad \boldsymbol{\Sigma}=\left(\begin{array}{cc}
0.1 & 0.06 \\
0.06 & 0.05
\end{array}\right) \\
\operatorname{Var}\left(\widetilde{r}_{C A P M}\right)=\left(\begin{array}{cc}
a & (1-a)) \\
= & 0.03 a^{2}+0.02 a+0.05 \\
0.06 & 0.05
\end{array}\right)\left(\begin{array}{cc}
a \\
1-a
\end{array}\right) \\
\sigma\left(\widetilde{r}_{C A P M}\right)=\sqrt{0.03 a^{2}+0.02 a+0.05} \\
\left.\frac{\partial \sigma\left(\widetilde{r}_{C A P M}\right)}{\partial a}\right|^{2}=\frac{1}{2} \frac{0.06 a+0.02}{\sqrt{0.03 a^{2}+0.02 a+0.05}} \\
\left.\frac{\partial \sigma\left(\widetilde{r}_{C A P M}\right)}{\partial a}\right|_{a=0}=\frac{1}{2} \frac{0.02}{\sqrt{0.05}} \\
\left.\frac{\partial E\left(\widetilde{r}_{C A P M}\right)}{\partial \sigma\left(\widetilde{r}_{C A P M}\right)}\right|_{a=0}=\frac{0.0447}{0.0447}=2.24
\end{gathered}
$$


Definizione 11 Equilibrio ("per gli oggetti di scelta").

Ponendo la pendenza dell'insieme nel punto di equilibrio di mercato (M) (??) pari alla pendenza della "capital market line" rispetto al portafoglio di tangenza (??)

$$
\frac{E\left(\tilde{r_{M}}\right)-r_{f}}{\sigma_{M}}=\frac{E\left(\tilde{r_{k}}\right)-E\left(\tilde{r}_{M}\right)}{\left(\sigma_{k, M}-\sigma_{M}^{2}\right) / \sigma_{M}}
$$

si trova il rendimento d'equilibrio commisurato al rischio dell'attività $i$ - esima, affinché essa sia domandata nel portafoglio di mercato: questa è l'equazione del $C A P M$ :

$$
\begin{gathered}
E\left(\tilde{r_{k}}\right)=r_{f}+\frac{\sigma_{k M}}{\sigma_{M}^{2}}\left[E\left(\tilde{r_{M}}\right)-r_{f}\right]= \\
=r_{f}+\beta_{k M}\left[E\left(\tilde{r_{M}}\right)-r_{f}\right]
\end{gathered}
$$

E' l'equazione di una retta che viene detta "security market line"se vista nel piano $\left[E\left(\tilde{r}_{k}\right), \beta_{k M}\right]$,

La stessa equazione si può scrivere anche come

$$
E\left(\tilde{r_{k}}\right)=r_{f}+\frac{\left[E\left(\tilde{r_{M}}\right)-r_{f}\right]}{\sigma_{M}} \frac{\sigma_{k M}}{\sigma_{M}}
$$

dove

(i) $\left[E\left(r_{M}\right)-r_{f}\right] / \sigma_{M}$ rappresenta il prezzo del rischio di mercato e

(ii) $\sigma_{k M} / \sigma_{M}$ la quantità di rischio appartenente all'attività $i$ - esima.

Tuttavia nell'uso comune si usa sempre la (17) e il prezzo del rischio viene misurato come correlazione all'eccesso di rendimento richiesto sull'attività rischiosa, misurato con $\beta_{i m}=\sigma_{k M} / \sigma_{M}^{2}$.

Fin qui abbiamo derivato un equilibrio simile all'equilibrio del produttore del metodo generale, ovvero e' stato risposto alla domanda: qual'e' il rendimento (simil prezzo d'offerta) ottimo in equilibrio per un'attività che ha un certo rischio in proporzione al rischio del mercato. Questa e' una sorta di curva d'offerta per le attività. L'idea e' che le attività sono disponibili in quantità perfettamente elastica (curva d'offerta orizzontale) per cui la sola cosa rilevante e' la decisione sul rendimento da offrire per venderla sul mercato. Il CAPM mostra qual'è il rendimento sensato per un'attività rischiosa, dato il rischio di mercato.

\subsection{Proprietà del CAPM}

1. Il modello è di equilibrio parziale. Le quantità scambiate sono già percepite come di "equilibrio" e i prezzi sono d'equilibrio. L'unica domanda è sul portafoglio da detenere.

2. Il CAPM risponde alla domanda "quale deve essere il $E r_{i}$ deve essere correlato al mercato affinché i prezzi di equilibrio siano quelli di mercato osservati. (Stima del "beta").

3. (Danthine Donaldson 2015) pp. 212) La Capital Market Line ci dà la frontiera efficiente di distribuzione del portafoglio fra l'indice e il risk-free. Ma allora cosa possiamo dire di un'altra attività qualunque che non appartiene alla frontiera efficiente?

Partiamo dalla Security market line in cui $\beta_{i m}$ è la variabile dipendente

$$
E \widetilde{r}_{i}=r_{f}+\left[E \widetilde{r}_{m}-r_{f}\right] \beta_{i m}
$$

dove

$$
\beta_{i m}=\frac{\sigma_{i m}}{\sigma_{m}^{2}}
$$

e si moltiplica e divide il termine a destra per $\sigma_{m}$

$$
E \widetilde{r}_{i}=r_{f}+\left[\left(\frac{E \widetilde{r}_{m}-r_{f}}{\sigma_{m}}\right) \beta_{i m}\right] \cdot \sigma_{m}
$$


la SML si trasforma in una relazione in cui $\beta_{i m}$ va a "pesare" il rischio di mercato che l'attività i-esima deve considerare e la variabile indipendente diventa il rischio di mercato.

Inoltre

$$
\begin{aligned}
E \widetilde{r}_{i} & =r_{f}+\left[\left(\frac{E \widetilde{r}_{m}-r_{f}}{\sigma_{m}}\right) \beta_{i m}\right] \cdot \sigma_{m} \\
& =r_{f}+\left[\frac{E \widetilde{r}_{M}-r_{f}}{\sigma_{m}}\right] \frac{\sigma_{i m}}{\sigma_{m}^{2}} \cdot \sigma_{m}
\end{aligned}
$$

ma

$$
\sigma_{i m}=\rho_{i m} \sigma_{i} \sigma_{m}
$$

dunque

$$
\begin{aligned}
E \widetilde{r}_{i} & =r_{f}+\left(\frac{E \widetilde{r}_{M}-r_{f}}{\sigma_{m}}\right) \frac{\left(\rho_{i m} \sigma_{i} \sigma_{m}\right)}{\sigma_{m}^{2}} \cdot \sigma_{m} \\
& =r_{f}+\left(\frac{E \widetilde{r}_{M}-r_{f}}{\sigma_{m}}\right) \frac{\left(\rho_{i m} \sigma_{i}\right)}{\sigma_{m}^{2}} \cdot \sigma_{m}^{2} \\
& =\left(\text { semplificando } \sigma_{m}^{2}\right) \\
& =r_{f}+\left[\left(\frac{E \widetilde{r}_{M}-r_{f}}{\sigma_{m}}\right) \rho_{i m}\right] \cdot \sigma_{i}
\end{aligned}
$$

Nella (18) la lezione del CAPM è che soltanto la frazione $\rho_{i m}$ del rischio di mercato è remunerata dal mercato. Il resto $\left(1-\rho_{i m}\right)$ è "diversified away" quando l'attività è inserita nel portafoglio di mercato. Infatti

$$
\sigma_{m}=\sum_{i=1}^{N} w_{i}\left(\rho_{i m} \sigma_{m}\right)
$$

4. In equilibrio ogni attività deve avere un prezzo in modo che il suo rendimento aggiustato per il rischio sia sulla "security market line".

Questo implica che attività interne all'insieme, ovvero inefficienti, siano sulla retta. Perche'? Perche' non tutta la varianza e' rilevante per gli investitori. La varianza che non dipende dalla variabilità di $m$ e' diversificabile. Gli emittenti sono quindi disposti a pagare un premio al rischio per la sola covarianza con $m$.

$$
\text { Rischio totale }=\text { rischio sistematico }+ \text { rischio asistematico }
$$

Sia

$$
\tilde{R}_{i}-r_{f}=a_{i}+\beta_{i}\left(\tilde{R_{m}}-r_{f}\right)+\varepsilon_{i}
$$

e la varianza si possa scomporre in

$$
\begin{aligned}
\sigma_{i}^{2} & =\beta_{i}^{2} \sigma_{m}^{2}+\sigma_{\varepsilon}^{2} \\
& =\underbrace{\left(\rho_{\text {im }} \sigma_{i}\right)^{2}}_{\text {sistematico }}+\underbrace{\sigma_{\varepsilon}^{2}}_{\text {diversificabile }}
\end{aligned}
$$

quello diversificabile sparisce diversificando il portafoglio.

5. La misura del rischio per singole attività è additiva. Si può derivare che (cfr. Copeland-Weston)

$$
\beta_{P}=a \beta_{x}+(1-a) \beta_{y}
$$

Si veda la Critica di Roll (1977). 


\section{References}

[1] Brunnermeier, M. K. (2001). Asset pricing under asymmetric information: Bubbles, crashes, technical analysis, and herding. Oxford University Press.

[2] Campbell, J. Y. (2017). Financial Decisions and Markets: a Course in Asset Pricing. Princeton University Press.

[3] Copeland, T., Weston, J., \& Shastri, K. (2005). Financial theory and corporate policy. 1000 p. Editorial Pearson Addison Wesley, New York, USA

[4] Danthine, J. P., \& Donaldson, J. B. (2015). Intermediate Financial Theory. Academic Press. 3rd Ed.

[5] De Matos, J. A. (2001). Theoretical foundations of corporate finance. Princeton University Press.

[6] Elton, E. J., Gruber, M. J., Brown, S. J., \& Goetzmann, W. N. (2013). Modern portfolio theory and investment analysis. John Wiley \& Sons. 9th ed

[7] Grinold, R. C., \& Kahn, R. N. (2000). Active Portfolio Management. McGraw-Hill.

[8] Ingersoll, J. E. (1987). Theory of financial decision making (Vol. 3). Rowman \& Littlefield.

[9] Roll, R. (1977) A Critique of the Asset Pricing Theory's Tests', Journal of Financial Economics, Vol. 4, No. 2. 